\title{
Analysis of the reliability of smartphone-based goniometric applications
}

\author{
Mariana Saori Barboza Sakata ${ }^{1}$, Talita Wassmuth ${ }^{1}$, Luiz Alfredo Braun Ferreira ${ }^{2}$, Luciano Pavan Rossi ${ }^{1}$.
}

${ }^{1}$ Universidade Estadual do Centro-Oeste - Unicentro, Guarapuava (PR), Brazil; ${ }^{*}$ Faculdade Guairacá e Universidade Estadual do Centro-Oeste - Unicentro, Guarapuava (PR), Brazil

\begin{abstract}
Background: The goniometric applications of smartphones are accessible and portable tools that, through internal sensors, are able to mea sure joint movement. However, every instrument used in clinical practice must be valid and reliable, maintaining consistency in its values when subjected to the same variables, circumstances and subjects. Objective: Analyze the angular measurement of different smartphone-based goniometric applications, with different usage times and operating systems. Methods: The present study analyzed the reliability of smartphone applications for goniometry by measuring the angles of a polygon designed for the study using 100 smartphones and correlating different aspects and conditions of the devices. Results: In general, there were no differences for almost all the variables analyzed when compared to each other, there was only difference when comparing the data collected with the reference values. Conclusion: The variables analyzed in this study did not influence the angular readings, presenting minimal variations in the angles for all conditions.

Keywords: Joint Goniometry; Joint Range of Motion; Mobile Apps.
\end{abstract}

\section{BACKGROUND}

Range of motion (ROM) assessment, in addition to analyzing joint integrity and limitations, is essential in the therapist's daily clinical practice ${ }^{(1)}$. Present at first as an indispensable part of the physical examination, it is possible for it to quantitatively monitor the effectiveness and progression of the physiotherapeutic intervention, making it necessary for the formulation of a treatment protocol and emphasizing the crucial accuracy of the angular readings ${ }^{(2)}$. As an instrument used to establish these data, there is the universal goniometer (UG). It is a tool most used by therapists, considered gold standard, low cost, with easy access, portable and non-invasive ${ }^{(3)}$. For handling the UG, the professional needs to use both hands to maintain the stability of the limb and the correct positioning of the instrument ${ }^{(4)}$. It is essential to know the location of bone accidents and estimate the center of rotation of the evaluated joints, and it is difficult to perform, which can result in an inaccurate reading of the angles ${ }^{(5)}$. Through technological innovations, tools for angular measurements emerge through the use of mobile applications, developed in order to facilitate daily activities, assisting and performing specific tasks ${ }^{(6)}$.

These tools use smartphone resources to improve their functions, for example, the internal sensors i.e. accelerometer, camera and gyroscope, which make it possible to identify the position of the device in space, and thus use it in the health area for evaluation of ROM of joints ${ }^{(7,8)}$. Healthcare applications have already reached 500 million out of a total of 1.4 billion smartphone users in $2015^{(9)}$. Its use has been gaining popularity in academic research, and like any instrument used in clinical practice, it must be properly tested and validated (10), demonstrating its reliability, which can be understood by the consistency of a measure acquired through an instrument, susceptible to the same conditions, variables and subject $(11,12)$. For this reason, several studies have been evaluating the reliability, inter and intra examiners, testing the use of different goniometric applications in different joints of the human body ${ }^{(1,5,8,13-18)}$.

However, studies analyzing only goniometric applications, correlating aspects and conditions of the devices used, has not been found so far. Performing this assessment comparing whether there is a difference in angular measurements is of paramount importance to test the reliability of the smartphone and its ability to measure joint angles ${ }^{(5)}$. In this way, the objective of the study is to investigate the reliability of different applications for goniometry, as well as to verify if the time of use or the operating system of the smartphones is able to modify the angular measurements. 


\section{METHODS}

This is a cross-sectional study performed at the Physiotherapy Clinic-School of State University of the Midwest, UNICENTRO.

\section{Sample}

100 smartphones were evaluated, including IPhones ${ }^{\circledR}$, which operate through the "IOS" operating system (set of programs responsible for managing, storing and processing data from a given device) and other devices using the Android operating system, as shown in the table 1.

Table 1. Characteristics of the sample.

\begin{tabular}{llc} 
Operational system & Brands & Amount \\
\hline IOS & Iphone $\AA$ & 48 \\
& Samsung $\AA$ & 24 \\
ANDROID & Motorola $\AA$ & 16 \\
& Asus $\AA$ & 5 \\
& Lenovo $\AA$ & 5 \\
& LG® & 2 \\
\hline
\end{tabular}

\section{Instruments: Apparatus (3D Polygon)}

To obtain the angles, a non-regular quadrilateral standard model was built based on previous works ${ }^{(19)}$. This was developed through the Google SketchUp software version 8.0.14346 (Figure 1A), and its printing was done on a Cartesian 3D Printer, model Anet A8. The material used for making it was PLA (Lactic polyacid, figure 1B), a polymer obtained from a renewable and biodegradable vegetable material, which gives the $3 \mathrm{D}$ figure great rigidity and precision $^{(20)}$. These factors are indispensable, since the object needs to remain with the structure and angulation unchanged during several collections and constant handling, to keep the variable constant throughout the sample.

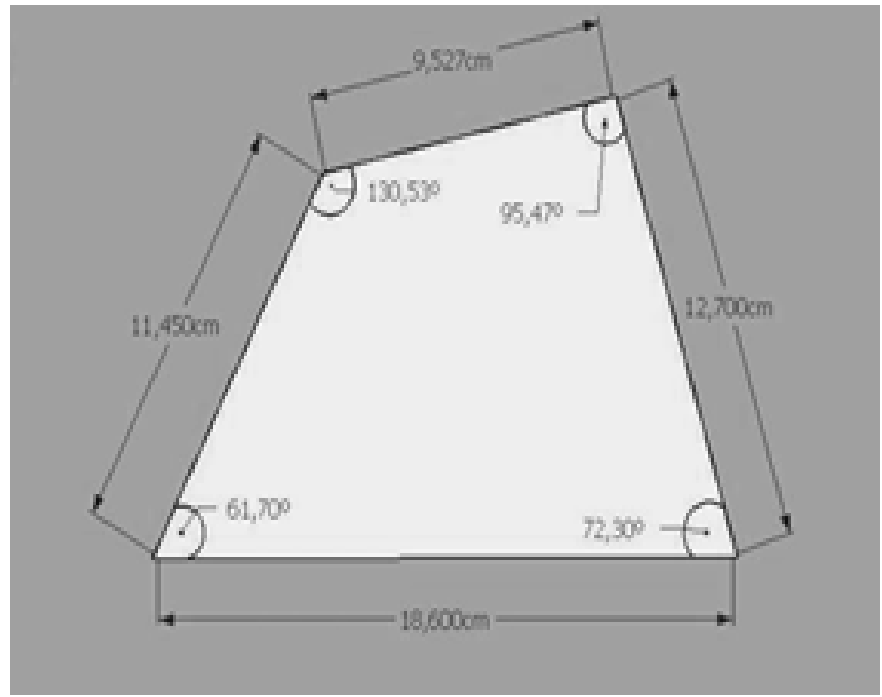

Figure 1A- Google SketchUp software project

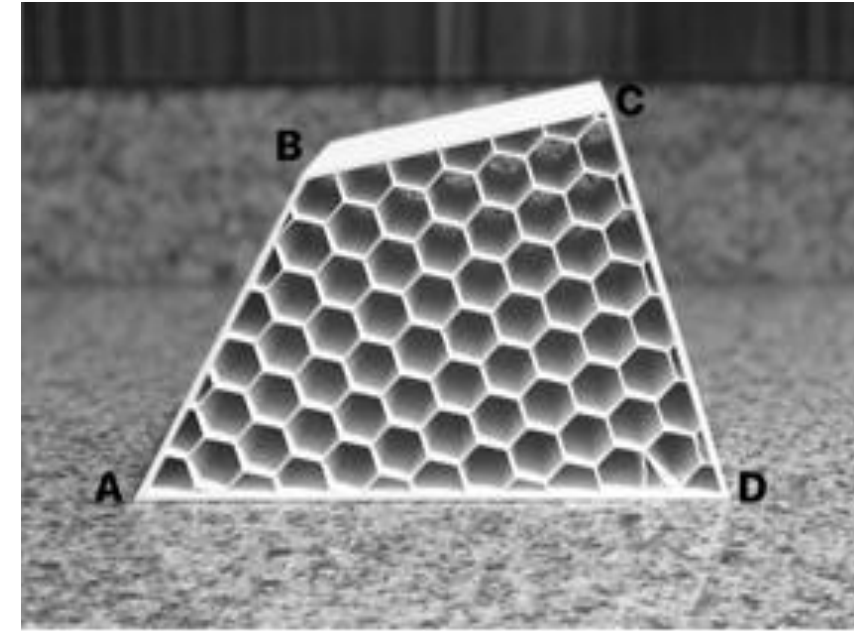

Figure 1B- 3D Prototype

"Note: Elaborated by the author, 2018.

To ensure the measurements of the polygon, high performance equipment (Mitutoyo Crysta Apex S 574) was used to measure the coordinates (Figure 2). The latter makes use of a tip that locates certain points of the object, transmitting the information to the computer, presenting its position and structure, making it possible to discover its angulation with accuracy.

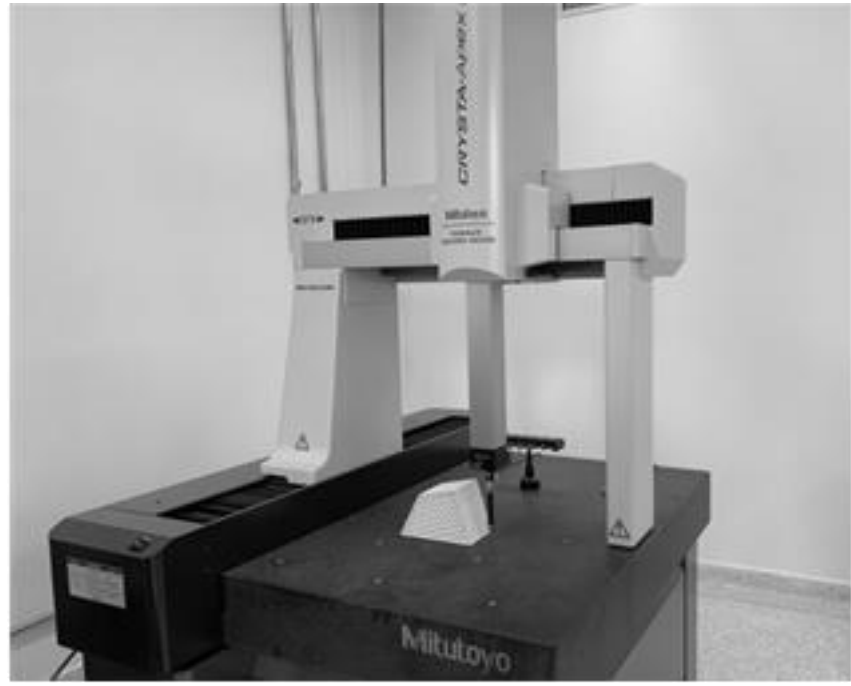

Figure 2- Mitutoyo Crysta Apex S 574

"Note: Elaborated by the author, 2019

\section{Application selection and usage}

After confirming the measurements through the Mitutoyo Crysta Apex S 574, the applications started to be selected through the digital distribution service Apple AppStore and Google Play. For this selection, all applications offered for free were analyzed, considering the feasibility of installation in the entire sample, and thus selecting those that had a clear understanding and better handling by the researchers. The selected applications were GoniometerPro ${ }^{\oplus}$, Angle Pro ${ }^{\oplus}$ and Angle Meter ${ }^{\oplus}$ for Apple smartphones, and Goniometer Records ${ }^{\oplus}$, Angle Pro ${ }^{\oplus}$ and Medidor de ângulos ${ }^{\circledast}$ for other brands. The GoniometerPro ${ }^{\circledR}$, 
Angle Pro ${ }^{\circledR}$ and Medidor de ângulos ${ }^{\circledast}$ have similar systems, in which it is enough to select the positioning of the device, given the support options on the surface evaluated with the lateral or posterior region of the phone, and thus position it over the location to be measured and the angle value will be described on the screen. The Angle Meter ${ }^{\circledast}$ has the same principle of use, but its measurements do not exceed $90^{\circ}$, due to this, on surfaces with an angle greater than this, it is necessary to add $90^{\circ}$ to the value indicated on the screen. In the case of the Goniometer Records ${ }^{\circledR}$, all measurements must be carried out with the support of the posterior region of the phone on the surface. First, it is necessary to lock in the starting position $\left(0^{\circ}\right)$, and then move the smartphone to the desired position, resulting in the value of the angle. However, in some situations the value that appears on the display is the supplementary angle to that observed, so it is necessary to perform a mathematical calculation, subtracting the value of $180^{\circ}$.

\section{Data collection}

Initially, it was necessary to obtain information regarding the phone, such as brand and time of use (less than one year, from one to three years and more than three years). The first step in measuring the angles was to remove the protective cover of the smartphone. Subsequently, one of the applications (all previously downloaded by the owner) was selected randomly. Then, the smartphone was aligned on the face of the polygon, and when positioned it remained in position (Figure 3 ) for 3 seconds, thus writing down the value provided on the display, except in applications where conversion was necessary. This execution was reproduced on the four faces, following the sequence $A B C D$ (Figure $1 B$ ), and when completed, the same process was perfomed in the next application. At the end of the analysis of the device, 12 measurements were collected.

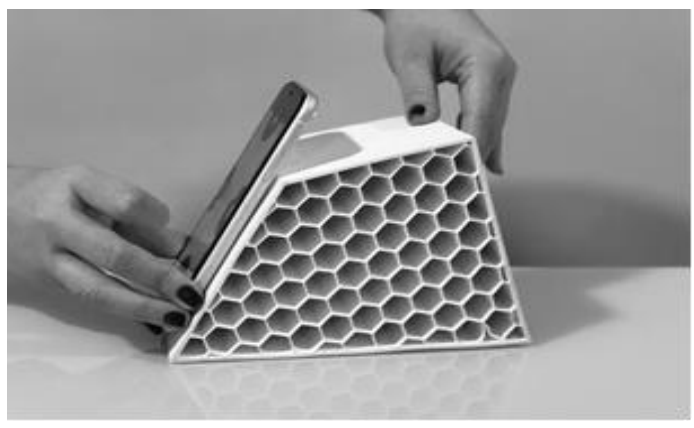

Figure 3- Positioning smartphone on the prototype *Note: Elaborated by the author, 2019

\section{Data analysis}

In order to examine the variation of the collected angular measurements, descriptive and inferential statistics were performed using the Statistica v.10 software for Windows.
So that the comparison of the collected data with the reference values of the polygon angles was possible, and as the applications read the integers, the four angles of the prototype were rounded: A: $61^{\circ}$; B: $131^{\circ}$; C: $95^{\circ}$; D: $73^{\circ}$. For the statistical analysis, the $D^{\prime}$ Agostino-Pearson normality test with parametric statistics were used. One-way ANOVA test was performed to verify the analysis of the variance of the angles according to the variables analyzed (application, operating system and time of use), being considered a level of significance in a $95 \%$ confidence interval. When there was a difference $(p<0.05)$ between the studied variables, the Sheffé test was used as post-hoc.

\section{RESULTS}

When analyzing the applications according to table 2, there were differences $(\mathrm{p}<0.05)$ in GoniometerPro ${ }^{\circ}$ 'Angle $\mathrm{Pro}^{\oplus}$, Angle Meter ${ }^{\circledast}$ and Medidor de ângulos ${ }^{\circledast}$ when compared to the standard value at angle C. Comparing the subgroups (columns) of the same angle, there was a difference between the Angle Pro ${ }^{\circledR}$ and the Goniometer Records ${ }^{\oplus}$, Angle Meter ${ }^{\oplus}$ and the Goniometer Records ${ }^{\oplus}$, Medidor de ângulos ${ }^{\oplus}$ and the Goniometer Records ${ }^{\oplus}$. As for angle D, the changes occurred only in the GoniometerPro ${ }^{\oplus}$, and Angle Pro ${ }^{\circledast}$ when compared to the standard.

Table 2. Angles (in degrees) obtained by goniometric applications.

\begin{tabular}{lllll}
\hline & Angle A & Angle B & Angle C & Angle D \\
\hline \multirow{4}{*}{ G PRO } & $61,13 \mathrm{a} \pm$ & $131,19 \mathrm{a} \pm$ & $94,31 \mathrm{bc} \pm$ & $73,77 \mathrm{a} \pm$ \\
& 1,02 & 0,98 & 1,15 & 1,21 \\
A PRO & $61,03 \mathrm{a} \pm$ & $131,36 \mathrm{a} \pm$ & $94,07 \mathrm{~cd} \pm$ & $73,60 \mathrm{a} \pm$ \\
& 1,10 & 1,26 & 1,34 & 1,15 \\
& $60,92 \mathrm{a} \pm$ & $131,04 \mathrm{a} \pm$ & $94,06 \mathrm{~cd} \pm$ & $73,46 \mathrm{a} \mathrm{b} \pm$ \\
A meter & 1,00 & 1,27 & 1,12 & 1,22 \\
& $61,08 \mathrm{a} \pm$ & $131,37 \mathrm{a} \pm$ & $94,94 \mathrm{ab} \pm$ & $73,40 \mathrm{a} \mathrm{b} \pm$ \\
G records & 1,25 & 2,06 & 1,65 & 1,42 \\
& $61,21 \mathrm{a}$ & $131,15 \mathrm{a} \pm$ & $94,12 \mathrm{~cd} \pm$ & $73,60 \mathrm{a} \mathrm{b} \pm$ \\
M angle & $\pm 1,30$ & 1,16 & 1,37 & 1,36 \\
& & & & \\
\multirow{2}{*}{ Pattern } & $61,00 \mathrm{a} \pm$ & $131,00 \mathrm{a} \pm$ & $95,00 \mathrm{a} \pm$ & $73,00 \mathrm{~b} \pm$ \\
& 0,00 & 0,00 & 0,00 & 0,00 \\
\hline
\end{tabular}

*Note: Means followed by different letters in the same column indicate a significant difference $(p<0.05)$ between one or more subgroups.

Related to the time of use of the device, Table 3 shows that significant differences were found in angles $C$ and $D$, in the three subgroups when compared to the standard value, but there is no difference when compared to each other. 
Table 3. Angles (in degrees) related to smartphone usage time.

\begin{tabular}{lcccc}
\hline & Angle A & Angle B & Angle C & Angle D \\
\hline Less than a year & $61,01 \mathrm{a} \pm 0.94$ & $131,39 \mathrm{a} \pm 1,21$ & $94,24 \mathrm{~b} \pm 1,21$ & $73,58 \mathrm{a} \pm 1,07$ \\
From 1 to 3 years & $61,19 \mathrm{a} \pm 1,11$ & $131,19 \mathrm{a} \pm 1,51$ & $94,41 \mathrm{~b} \pm 1,32$ & $73,66 \mathrm{a} \pm 1,35$ \\
More than 3 years & $60,98 \mathrm{a} \pm 1,36$ & $131,15 \mathrm{a} \pm 1,39$ & $94,11 \mathrm{~b} \pm 1,60$ & $73,45 \mathrm{a} \pm 1,34$ \\
Pattern & $61,00 \mathrm{a} \pm 0,00$ & $131,00 \mathrm{a} \pm 0,00$ & $95,00 \mathrm{a} \pm 0,00$ & $73,00 \mathrm{~b} \pm 0,00$ \\
\hline
\end{tabular}

${ }^{*}$ Note: Means followed by different letters in the same column indicate a significant difference $(p<0.05)$ between one or more subgroups

Regarding the operating system, there was no difference when compared to each other, however when compared to the standard in angles $C$ and $D$ there was a difference, as shown in the table 4.

Table 4. Angles (in degrees) related to the operating system.

\begin{tabular}{lcccc}
\hline & Angle A & Angle B & Angle C & Angle D \\
\hline IOS & $61,07 a \pm 1,03$ & $131,22 a \pm 1,15$ & $94,22 b \pm 1,14$ & $73,67 a \pm 1,20$ \\
Android & $61,06 a \pm 1,23$ & $131,28 a \pm 1,56$ & $94,31 b \pm 1,56$ & $73,48 a \pm 1,30$ \\
Pattern & $61,00 a \pm 0,00$ & $131,00 a \pm 0,00$ & $95,00 a \pm 0,00$ & $73,00 b \pm 0,00$ \\
\hline
\end{tabular}

Note: Means followed by different letters in the same column indicate a significant difference $(p<0.05)$ between one or more subgroups

\section{DISCUSSION}

The aim of this study was to analyze the angular measurement of different goniometric applications for smartphones, with different usage times and different operating systems. In general, there were no differences for almost all the variables analyzed when compared to each other, there was only differences when comparing the data collected with the reference values. It is also possible to notice that differences occurred only in the angles $\mathrm{C}\left(95^{\circ}\right)$ and $D\left(73^{\circ}\right)$, both in applications and in operating systems and time of use when compared to the reference value. This difference in angles $C$ and $D$ between the values of the goniometric application and the reference value for all variables was less than 1 , however, detected by the posthoc after performing the ANOVA test, it can be explained by different calibrations between the goniometric application and the high performance equipment to measure the coordinates and generate precise joint angles (Mitutoyo Crysta Apex S 574 ${ }^{\circ}$ ).

Another point to be considered is the proximity of the values to the $90^{\circ}$ angle, which implies that the smartphone has greater contact with the surface on which the polygon is supported, so that its angular reading can be influenced by the unevenness of it. This factor corroborates with the fact that the Goniometer Records ${ }^{\circ}$ was the only application that showed no difference in any of the angles (Table 2). An alternative raised is that in its use, the therapist who positions the measurement starting point $\left(0^{\circ}\right)$, regardless of the angle at which the surface is located, thus the inclination of the surface does not influence the support of the smartphone and its angle. This is of great importance in clinical practice, because when analyzing a joint, the starting position of the movement does not always coincide with the $0^{\circ}$ given by the application. Alba-Martín ${ }^{(1)}$ points out that the correct configuration of the application requires an active participation of the therapist, aligning the measurement axes with the baseline or reference of the application. When this alignment is not possible, it is necessary for the therapist to evaluate the value that appears on the display before the movement of the joint (initial position) of the value found after the range of motion (final position) and thus calculate the range of motion allowed by the joint. Another example is the Angle Meter ${ }^{\circ}$ used in the study, in which the maximum accepted angulation is $90^{\circ}$, directly implying the evaluation of joints that allow movements with angles greater than this, such as shoulder, elbow and knee flexion.

The results found are close to the studies of Kuegler et al. ${ }^{(19)}$, that present a similar methodology through the analysis of different applications and operating systems in an irregular pyramid, where the standard deviation of Apple and Samsung devices were less than 3o, in which the author recommends that the use of these applications is acceptable for practice clinic.

Hambly, Sibley, Ockendon ${ }^{(2)}$ add that small angular differences do not have an important clinical impact on the assessment of joint movement and suggest that the minimum clinically relevant measurable difference would be 5 o (21) to 10 일 ${ }^{(22)}$, showing that greater angular variations during a goniometric evaluation may be the result of an evaluator error ${ }^{(2)}$. Among the phones analyzed in the study, the variation in the data collected on the reference value did not exceed that described by the authors above, thus demonstrating that it would not cause clinical changes. Another point is the different applications evaluated. In 
their systematic review, Keogh et al. ${ }^{(23)}$ point out the diversity of applications used and their reliability, demonstrating that the therapist has several options, being able to choose the best application to analyze a particular joint.

Dos Santos et al. ${ }^{(24)}$ show that despite the functional difference between the applications, which use the inclination generated by the evaluated surface during the range of motion, and the universal goniometer, in which the angular measurement occurs through the movement of one of the instrument's arms on its axis, the smartphone proved to be reliable to the variation of the knee flexion angle compared to the goniometer. In addition, Milanese et al. ${ }^{(5)}$ demonstrate the ease of understanding and use of the applications, which the use of the it in the evaluation of the knee joint proved to be reliable for both trained evaluators and beginners. Studies of Pourahmadi et al. ${ }^{(8)}$ and Cox et al. ${ }^{(16)}$ bring as an advantage of goniometric applications the easy access, being able to be downloaded in all smartphones (however they differ in different operating systems), and available at any time and place.

However, this feature also has limitations in clinical practice, such as direct contact between the smartphone and the patient's skin ${ }^{(25)}$, increasing the risk of infection and proliferation of bacteria, unless properly covered, and the cost of purchasing a device for clinical use is high (18), standing out at the low cost of the universal goniometer. As they are technologies, they are exposed to constant evolution and transitions, due to the volatility of it. As well as they are subject to intrinsic factors of the device, for example the battery level or a software malfunction error (bug), which can cause an unexpected stop or interfere with the measurement ${ }^{(8)}$.

Its various applications exemplify its complexity, which are influenced by frequent progress in its physical components (hardware) and programs/systems responsible for its operation (software). Hanock, Hepworth, Wenbridge ${ }^{(18)}$ suggest that software and hardware changes lead to inherent errors. Relating this statement, the IOS operating system demonstrated a smaller standard deviation in all angles (table 4), showing that the data are less dispersed, thus presenting greater precision, despite not showing any significant difference in relation to the Android system. The same occurred when analyzing the time of use of the devices (table 3 ), in which devices with shorter time of use provided more accurate data, despite not showing a significant difference with longer times of use. However, precision does not necessarily correspond to being more accurate, since accuracy refers to the data being closer to the real value. There are limitations in the study, since a sample calculation was not performed to define the required sample size, despite the attempt to analyze a large number of smartphones. Finally, for the feasibility of the study, only free applications were used, and in future studies the verification of other applications could be performed.

\section{CONCLUSION}

There was no difference in all the variables analyzed when compared to each other, that is, different goniometric applications, different times of use and/or smartphone operating system were not able to modify the angular measurements through the evaluation system proposed in the study.

AUTHOR'S CONTRIBUTION: MSBS e TW developed the study and the corresponding text, as well as the data collection; LPR carried out the supervision and guidance of the study; LABF carried out the supervision and guidance of the study; LABF performed a critical intellectual review of the manuscript. All authors read and approved the final manuscript.

FUNDING: nothing to declare.

CONFLICT OF INTEREST: nothing to declare.

\section{REFERENCES}

1. Alba-Martín R. Reliability and validity of shoulder and elbow measurements: analysis of an Android application and a goniometer. Rehab. 2016;50(2):7174.

2. Hambly K, Sibley R, Ockendon M. Level of agreement between a novel smartphone application and a long arm goniometer for the assessment of maximum active knee flexion by an inexperienced tester. Int J of Physioth \& Rehab. 2012;(2).

3. Santos JDMD, Oliveira MAD, Silveira NJFD, Carvalho SDS, Oliveira AG. Inter and intra-examiner reliability in angular measurements by digital photogrammetry and goniometry. Fisiot em Mov. 2011;24(3):389-400.

4. Behnoush B, Tavakoli N, Bazmi E, Fard FN, Shahi MHP, Okazi $A$, et al. Smartphone and universal goniometer for measurement of elbow joint motions: a comparative study. Asian J of Sports Med. 2016;7(2):e30668.

5. Milanese S, Gordon S, Buettner P, Flavell C, Ruston S, Coe $D$, et al. Reliability and concurrent validity of knee angle measurement: smart phone app versus universal goniometer used by experienced and novice clinicians. Man Ther. 2014;19(6):569-574.

6. Mehta SP, Barker K, Bowman B, Galloway H, Oliashirazi $\mathrm{N}$, Oliashirazi A. Reliability, concurrent validity, and minimal detectable change for Iphone goniometer app in assessing knee range of motion. The J of Knee Surg. 2017;30(6):577-584.

7. Milani P, Coccetta CA, Rabini A, Sciarra T, Massazza G, Ferriero $G$. Mobile smartphone applications for body position measurement in rehabilitation: a review of goniometric tools. PM\&R. 2014;6(11):1038-1043. 
8. Pourahmadi MR, Takamjani IE, Sarrafzadeh J, Bahramian M, Mohseni-Bandpei MA, Rajabzadeh F, et al. Reliability and concurrent validity of a new iPhone goniometric application for measuring active wrist range of motion: a cross-sectional study in asymptomatic subjects. J of Anat. 2017;230(3):484495.

9. Ralf-Gordon J. 500 Million People Will be Using Mobile Health Applications in 2015. News from research2guidance. 2010.

10. Wellmon RH, Gulick DT, Paterson ML, Gulick CN. Validity and reliability of two goniometric mobile apps: device, application, and examiner factors. J of Sport Rehab. 2016;25(4):371-379.

11. Carvalho RMFD, Mazzer N, Barbieri CH. Reliability and reproducibility analysis of goniometry in relation to hand photogrammetry. Acta Orto Bras. 2012;20(3):139-149.

12. Dos Santos CM, Ferreira G, Malacco PL, Sabino GS, Moraes GFS, Felício DC. Intra and inter-examiner reliability and measurement error when using goniometer and digital inclinometer. Rev. Bras. Med. Esporte. 2012;18(1):38-41.

13. Otter SJ, Agalliu B, Baer N, Hales G, Harvey K, James $\mathrm{K}$, et al. The reliability of a smartphone goniometer application compared with a traditional goniometer for measuring first metatarsophalangeal joint dorsiflexion. J of Foot and Ankle res. 2015;8(1):30.

14. Johnson LB, Sumner S, Duong T, Yan P, Bajcsy R, Abresch RT, et al. Validity and reliability of smartphone magnetometer-based goniometer evaluation of shoulder abduction-a pilot study. Man Ther. 2015;20(6):777-782.

15. Cuesta-Vargas Al, Roldán-Jiménez C. Validity and reliability of arm abduction angle measured on smartphone: a cross-sectional study. BMC Musc Disord. 2016;17(1):93.

16. Cox RW, Martinez RE, Baker RT, Warren L. Validity of a smartphone application for measuring ankle plantar flexion. Journal of sport rehabilitation. 2018;27(3).

17. Furness J, Schram B, Cox AJ, Anderson SL, Keogh J. Reliability and concurrent validity of the iPhone ${ }^{\circledR}$ Compass application to measure thoracic rotation range of motion (ROM) in healthy participants. Peer J. 2018;6:e4431.

18. Hancock GE, Hepworth T, Wembridge K. Accuracy and reliability of knee goniometry methods. J of Exp Ortho. 2018;5(1):46.

19. Kuegler P, Wurzer P, Tuca A, Sendlhofer G, Lumenta $D B$, Giretzlehner $M$, et al. Goniometer-apps in hand surgery and their applicability in daily clinical practice. Saf in Health. 2015;1(1):11.
20. Bieging P. Art, New Technologies and Communication: Contemporary Phenomenology. São Paulo: PMS Comun e Design. 2010;288-292.

21. Chaudhary R, Beaupre LA, Johnston DW. Knee range of motion during the first two years after use of posterior cruciate-stabilizing or posterior cruciateretaining total knee prostheses. A randomized clinical trial. J Bone Joint Surg Am. 2008;90(12):2579-2586.

22. Jakobsen TL, Christensen $M$, Christensen SS, Olsen $M$, Bandholm T. Reliability of knee joint range of motion and circumference measurements after total knee arthroplasty: does tester experience matter? Physiother Res Int. 2010;15(3):126-134.

23. Keogh JW, Cox A, Anderson S, Liew B, Olsen A, Schram $B$, et al. Reliability and validity of clinically accessible smartphone applications to measure joint range of motion: A systematic review. PloS one. 2019;14(5):e0215806.

24. Dos Santos RA, Derhon V, Brandalize M, Brandalize D. Rossi LP. Evaluation of knee range of motion: Correlation between measurements using a universal goniometer and a smartphone goniometric application. J of Bodyw and Mov Ther. 2017;21(3):699-703.

25. Salamh PA, Kolber $M$. The reliability, minimal detectable change and concurrent validity of a gravity-based bubble inclinometer and Iphone application for measuring standing lumbar lordosis. Physiother theo and pract. 2014;30(1):6267. 